[Communication]

\title{
The Novel Application of Polyclonal Antibodies in Recognizing Anti-EGFR Inhibitors Directly from Herb *
}

\author{
Zhu Li-Li Xu Xiao-Jie \\ ( College of Chemistry and Molecular Engineering, Peking University, Beijing 100871)
}

\begin{abstract}
A polyclonal antibody (PcAb) prepared with piceatannol, a known inhibitor against the epidermal growth factor receptor (EGFR), was adopted as a stationary phase in the chromatographic system. Using the antibody to mimic the enzyme, several anti-EGFR inhibitors were recognized directly from a crude extract of Tibetan herb. Frontal affinity chromatography (FAC) was used here for analyzing molecular interactions between the analytes and an immobilized ligand (in this case the PcAb conjugated to Sepharose CL-4B) by calculating the extent of retardation of the elution front. By combining FAC with mass spectrometry (MS) in the current study, a very efficient and straightforward procedure was developed for analyzing the binding properties of different inhibitors. The novel effective screening of anti-EGFR inhibitors using PcAb from natural resources affords us a new feasible approach for the discovery of lead compounds.
\end{abstract}

Keywords: Epidermal growth factor receptor (EGFR), Polyclonal antibody (PcAb), Inhibitor, Frontal affinity chromatography (FAC)-electrospray ionization mass spectrometry (ESI-MS)

The epidermal growth factor receptor (EGFR) belongs to the class of the trans-membrane growth factor receptor PTK' $s$ and it plays a fundamental role in the regulation of cell growth ${ }^{[1]}$. However, under certain conditions, as a result of over-expression, mutation or co-expression of the ligand and the receptor, EGFR can become hyper-activated; the result of this is uncontrolled cell proliferation ${ }^{[2-4]}$. The EGFR is known to be over-expressed in a large percentage of clinical cancers of various types (including those derived from the brain, lung, ovarian, bladder, head and neck) and to be associated with poor prognosis ${ }^{[5-7]}$. Compounds those can specifically inhibit the tyrosine kinase activity of EGFR are therefore of potential interests as anticancer ligands, and this is an active field of drug discovery.

In our previous work ${ }^{[8]}$, a known anti-EGFR inhibitor, (E) -piceatannol, was extracted from Caragana Jubata by a solid-phase extraction procedure with a molecular imprinted polymer (MIP) as the sorbent material. In this study, a polyclonal antibody $(\mathrm{PcAb})$ raised against piceatannol, which was linked with bovine serum albumin (BSA), was covalently bound to the chromatographic packing for mimicking the enzyme. Several anti-EGFR inhibitors including piceatannol itself were recognized from the extract of Caragana Jubata, a traditional Tibetan medicine. Herein, frontal affinity chromatography (FAC) connected with mass spectrometry (MS) was adopted as the analytical method. The efficient application of PcAb as the enzyme alternative to recognize small molecular inhibitors from natural resources has rarely been reported. It affords us a novel and straightforward approach for the screening of novel inhibitors, which would be very beneficial for the discovery of lead compounds.

\section{Experimental}

\section{1 Chemicals and Materials}

(E) -piceatannol (3, 5, 3', 4'-tetrahydroxy-stilbene) (1) and other five compounds studied in this work were isolated from Caragana Jubata by our group. And their anti-EGFR inhibitory activities were evaluated by ELISA assay according to the literature ${ }^{[9]}$. The structures of these analytes were shown in Fig. 1. Mixtures of polyclonal antibodies against piceatannol were produced by immunization of rabbits with piceatannol conjugated to bovine serum albumin (BSA). Two white male New Zealand rabbits were obtained from Life Science Center, Peking University. BSA, ovalbumin (OVA) and Sepharose CL-4B were bought from Sigma Bioscience (USA). The water soluble

Received: February 25, 2003; Revised: March 18, 2003. Correspondent: Xu Xiao-Jie (E-mail: xiaojxu@ chem. pku. edu. cn). "The Project Supported by NSFC (29992590-2) and the 211 Foundation of Peking University 


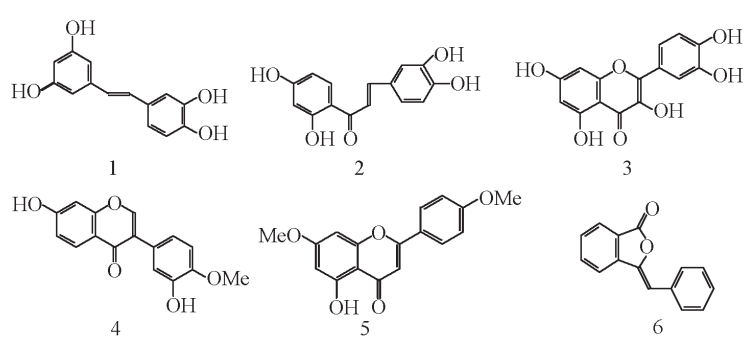

Fig. 1 The chemical structures of the compounds studied in this work

1-ethyl-3 (3-dimethylaminopropyl) -carbodiimide hydrochloride (EDCI) was purchased from Sigma-Aldrich (Germany). Freund's complete adjuvant and incomplete adjuvant were purchased from Gibco.

\section{2 Preparation of the polyclonal antibodies}

The compound piceatannol was modified and then linked to bovine serum albumin (BSA) before immunization. Because there are 4 hydroxyl groups in the piceatannol molecule, anyone of them could be reacted with $\mathrm{ClCH}_{2} \mathrm{COONa}$ and modified to the corresponding carboxyl derivative by controlling the quantity of the reagent: the ratio between $\mathrm{ClCH}_{2} \mathrm{COONa}$ and piceatannol was less than 1. And then the carboxyl derivative of piceatannol was linked to BSA with the activation of EDCI. The final ratio of the coupling between piceatannol and BSA was 14: 1 .

Immune two white male New Zealand rabbits every 10 days for 4 times using the antigen (piceatannol linked with BSA). After the fourth immunity, both of the rabbits had been phelbotomized from ears. ELISA test showed the potency of the serum was 80000 . The blood was collected from arteria of rabbit's neck one week after the last immunity. There were totally about $50 \mathrm{~mL}$ serums collected from the two rabbits. The antibody was isolated through the sedimentation of saturation ammonium sulfate (SAS) solution at first, and then was purified through an affinity column of BSA. There were totally 3 times for the sedimentation procedure: the saturation of the solution was $40 \%$ for the first two times and $35 \%$ for the last one. The potency of the purified antibody was 16000 .

After purification, the antibodies were immobilized to Sepharose CL-4B beads by the activation of epichlorohydrin. The concentration of protein in the mixture after immobilization was $4.8 \mathrm{~g} \cdot \mathrm{L}^{-1}$, and the final volume was $3.2 \mathrm{~mL}$. There were totally about $2.4 \mathrm{~g}$ of wet Sepharose CL-4B beads $(3.5 \mathrm{~mL})$ linked with $15.4 \mathrm{mg}$ of the purified antibodies. Using a self-pack device to pack the affinity columns $(\phi \times l=2.1 \mathrm{~mm} \times 30 \mathrm{~mm})$ with the polyclonal antibodies conjugated covalently to Sepharose CL-4B beads. $100 \mu \mathrm{L}$ of wet Sepharose beads that had been linked with PcAb was filled in a column. Then the PcAb columns were equilibrated with $2 \mathrm{mmol} \cdot \mathrm{L}^{-1} \mathrm{NH}_{4} \mathrm{Ac}$ solution ( $\mathrm{pH}$ 6. 7 )

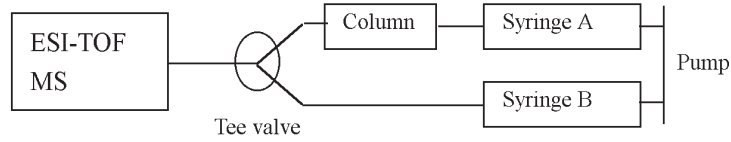

Fig. 2 System diagram of the frontal affinity chromatography-mass spectrometry

The analyte solution in syringe A and the solvent methanol in syringe $\mathrm{B}$ are mixed in the tee valve and then detected by ESI-TOF MS.

and kept at $4{ }^{\circ} \mathrm{C}$.

\section{3 Preparatiorfothextractample}

Air-driedrootso CaraganaJubatawerextractedbymacerating with $85 \%$ ethanol at room temperature. The solvent was evaporated in vacuum and then was partitioned between $\mathrm{H}_{2} \mathrm{O}$, petroleum ether, $\mathrm{CHCl}_{3}, \mathrm{EtOAc}$ and $n$-butanol successively. ELISA assays suggested that the $\mathrm{CHCl}_{3}$ and EtOAc extracts were efficient in inhibiting the tyrosine kinase activity of EGFR. Therefore, in the following study, the mixture of these two extracts of Caragana Jubata was investigated. The extract sample (dry weight, $5 \mathrm{mg}$ ) was evaporated to dryness in a vacuum centrifuge (RE 52A, Shanghai, China) and then re-dissolved in $1 \mathrm{~mL}$ methanol to be qualified as $5 \mathrm{~g} \cdot \mathrm{L}^{-1}$, thereafter it was diluted to be $0.1 \mathrm{~g} \cdot \mathrm{L}^{-1}$ with $2 \mathrm{mmol} \cdot \mathrm{L}^{-1} \mathrm{NH}_{4} \mathrm{Ac}(\mathrm{pH}$ 6. 7 ) before loading on the $\mathrm{PcAb}$ column.

\section{4 Frontal affinity chromatography-mass spectrom- etry}

The frontal affinity chromatographic experiments were done at room temperature at a flow rate of $10 \mu \mathrm{L} \cdot \mathrm{min}^{-1}$ in the running buffer. The device was designed as shown in Fig. 2. The antibodies were immobilized to Sepharose CL-4B and then packed wetly in the affinity column. Syringes A and B are $2.5 \mathrm{~mL}$ : A contains the solution of the sample in $2 \mathrm{mmol} \cdot \mathrm{L}^{-1} \mathrm{NH}_{4} \mathrm{Ac}$ while $\mathrm{B}$ contains methanol, and these two solutions are mixed in the tee valve and then enter the detector of ESI-MS. A Mariner electrospray ionization time-of-flight (ESI-TOF) mass spectrometer (PE Biosystems, USA) was used to analyze the sample. For ESI, the mass spectrometer was operated in the negative mode using the following conditions: spray tip potential, $5000 \mathrm{~V}$; nozzle potential, $100 \mathrm{~V}$; detector voltage, $1950 \mathrm{~V}$; nozzle temperature, 140 ${ }^{\circ} \mathrm{C}$; Quad temperature, $140{ }^{\circ} \mathrm{C}$. All the other parameters were set as default values.

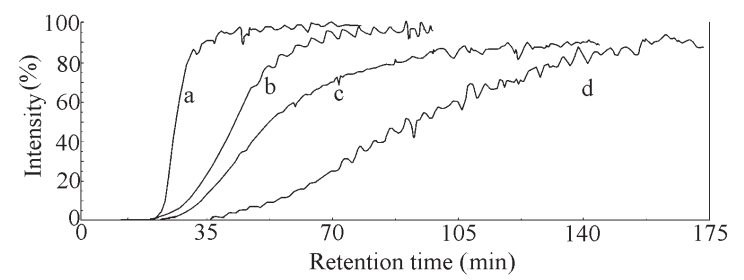

Fig. 3 Frontal affinity chromatograms of the solutions of the analyte (piceatannol) with different concentrations $c / \mathrm{mg} \cdot \mathrm{L}^{-1}:$ a) 20, b) 10, c) 5, d) 2.5 


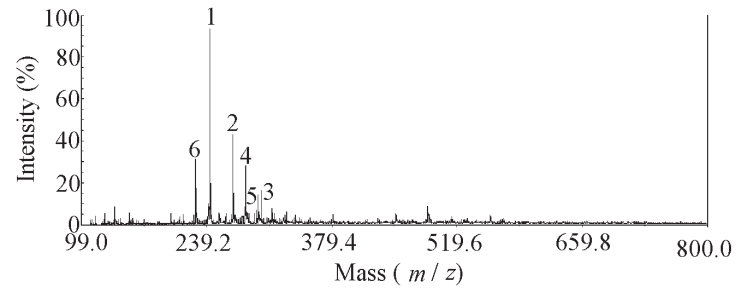

Fig. 4 The mass spectrum of the extract sample of Caragana Jubata

The six compounds displayed in Fig. 1 were corresponding to the six main peaks in this spectrum

\section{Results and discussion}

\section{1 Determination of ligand content, $L_{t}$, and dissoci-} ation constant, $\boldsymbol{K}_{\mathrm{d}}$

Frontal affinity chromatographic analysis of the binding interaction between piceatannol and the PcAb revealed the specific affinity of the absorbent to the analyte. Equation (1) is the basic equation of frontal affinity chromatography:

$$
V-V_{0}=\frac{L_{\mathrm{t}}}{c+K_{\mathrm{d}}}
$$

It also can be put in the following form:

$$
\frac{1}{c\left(V-V_{0}\right)}=\frac{1}{L_{\mathrm{t}}}+\frac{K_{\mathrm{d}}}{L_{\mathrm{t}}} \cdot \frac{1}{c}
$$

where $V$ is the elution volume of the analyte; $c$ is the concentration of the analyte; $V_{0}$ is the elution volume of a controlled substance having no affinity (Sepharose CL-4B in the present work); $L_{\mathrm{t}}$ is the total amount of immobilized ligand; and $K_{\mathrm{d}}$ is the dissociation constant.

We applied the present FAC-MS to test the binding specificity of the PcAb to the original half-antigen, piceatannol. 2. 5 $\mathrm{mL}$ solution of different concentrations ranged from $20 \mathrm{mg} \cdot \mathrm{L}^{-1}$, $10 \mathrm{mg} \cdot \mathrm{L}^{-1}, 5 \mathrm{mg} \cdot \mathrm{L}^{-1}$ to $2.5 \mathrm{mg} \cdot \mathrm{L}^{-1}$ of piceatannol in 2 mmol $\cdot \mathrm{L}^{-1} \mathrm{NH}_{4} \mathrm{Ac}$ was filled in syringe A (as shown in Fig. 2) and impelled through the $\mathrm{PcAb}$ column, while $2.5 \mathrm{~mL}$ of methanol was filled in syringe $\mathrm{B}$ and pushed forward synchronously with A at the rate of $10 \mu \mathrm{L} \cdot \mathrm{min}^{-1}$. A total-ion chromatography (TIC) was obtained for 200-minute run time for the mixed solution. The selected-ion chromatography of piceatannol that derived from TIC gives the frontal time (Fig. 3). And the corresponding results were shown in Table 1.

From the four traces corresponding to different concentra-

Table 1 Results of frontal affinity chromatography of piceatannol

\begin{tabular}{ccccc}
\hline$\frac{c}{\mathrm{mg} \cdot \mathrm{L}^{-1}} \frac{c}{\mu \mathrm{mol} \cdot \mathrm{L}^{-1}}$ & & $\frac{c}{\mu \mathrm{mol}^{-1} \cdot \mathrm{L}}$ & $V-V_{0} / \mu \mathrm{L}$ & $\frac{\left[c\left(V-V_{0}\right)\right]^{-1}}{\mu \mathrm{mol}^{-1}}$ \\
\hline 20.0 & 82.0 & 0.0122 & 188.0 & 64.9 \\
10.0 & 41.0 & 0.0244 & 314.0 & 77.7 \\
5.0 & 20.5 & 0.0488 & 486.0 & 100.4 \\
2.5 & 10.2 & 0.0976 & 676.0 & 144.9 \\
\hline
\end{tabular}

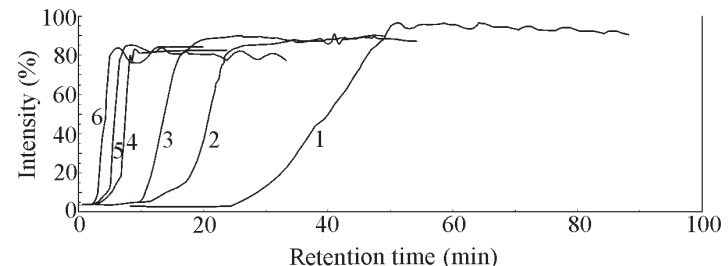

Fig. 5 The selected-ion FAC trace for six analytes existed in the extract sample of Caragana Jubata The six traces for $1 \sim 6$ were corresponding to the six compounds shown in Fig. 1.

tions of the analyte, the ligand content, $L_{\mathrm{t}}$, and dissociation constant, $K_{\mathrm{d}}$ could be calculated, which were $44.90 \mathrm{mg} \cdot \mathrm{L}^{-1}$ and 17. $14 \mu \mathrm{mol} \cdot \mathrm{L}^{-1}$, respectively. From the data it could be concluded that the PcAb possessed obvious specific affinity to the half-antigen, piceatannol.

\section{2 The binding study of the PcAb for several an- ti-EGFR inhibitors in the crude extract sample}

Since the main object of the current work was to study the feasibility of using PcAb to mimic the receptor or enzyme in recognizing the anti-EGFR inhibitors directly from the crude extract of Caragana Jubata, the FAC-MS procedure was performed on $2.5 \mathrm{~mL}$ solution of the extract sample with the concentration of $100 \mathrm{mg} \cdot \mathrm{L}^{-1}$ in $2 \mathrm{mmol} \cdot \mathrm{L}^{-1} \mathrm{NH}_{4} \mathrm{Ac}$. The frontal affinity chromatographic trace derived from the mass spectra (Fig. 4) was shown in Fig. 5. Table 2 listed the results of the PcAb column for several main analytes existed in the extract sample.

From the data shown in Table 2 it could be seen the efficiency for the PcAb in recognizing different anti-EGFR inhibitors. According to the frontal volume and approximate concentration of each compound mainly existed in the extract, the corresponding dissociation constants $\left(K_{\mathrm{d}}\right)$ for the six main analytes were calculated, which were contrasted to their anti-EGFR inhibitory activities in Table 3 . From the data shown in Table 3 it could be observed that the higher was the bioactivity of the analyte, the stronger was the binding affinity between the compound and the PcAb, which meant that the affinity column based on the

Table 2 Results of the FAC procedure for six main analytes in the extract sample

\begin{tabular}{cccccc}
\hline \#Comp. & $\begin{array}{c}\text { Comp. } \\
\text { Approx. } \\
\text { conc. }(\%)\end{array}$ & $t / \mathrm{min}$ & $V / \mu \mathrm{L}$ & $V-V_{0} / \mu \mathrm{L}$ & $\begin{array}{c}K_{\mathrm{d}} / \mathrm{mol} \cdot \mathrm{L}^{-1} \\
(\text { estimated })\end{array}$ \\
\hline 1 & 7.6 & 45.3 & 453 & 409 & $1.7 \times 10^{-5}$ \\
2 & 5.4 & 21.9 & 219 & 175 & $8.1 \times 10^{-5}$ \\
3 & 2.5 & 19.6 & 196 & 152 & $9.7 \times 10^{-5}$ \\
4 & 2.7 & 8.3 & 83 & 39 & $4.2 \times 10^{-4}$ \\
5 & 2.0 & 7.2 & 72 & 28 & $5.7 \times 10^{-4}$ \\
6 & 3.0 & 5.0 & 50 & 6 & $3.6 \times 10^{-3}$ \\
\hline
\end{tabular}

\#The six compounds shown in Fig. 1 
Table 3 The recognition effect of the PcAb expressed in the contrast

\begin{tabular}{|c|c|c|}
\hline "Comp. & Relative binding strength & $\mathrm{IC}_{50} / \mathrm{mol} \cdot \mathrm{L}^{-1}$ \\
\hline 1 & 1.00 & $4.9 \times 10^{-6}$ \\
\hline 2 & 0.21 & $1.0 \times 10^{-5}$ \\
\hline 3 & 0.18 & $1.5 \times 10^{-5}$ \\
\hline 4 & 0.04 & $1.1 \times 10^{-3}$ \\
\hline 5 & 0.03 & 1. $2 \times 10^{-3}$ \\
\hline 6 & 0.005 & $5.9 \times 10^{-2}$ \\
\hline
\end{tabular}

$\mathrm{PcAb}$ could recognize the inhibitors according to their bioactivities effectively. Especially for the three compounds $(1 \sim 3)$ that possess relatively higher inhibitory activities against EGFR, the recognition effect of the affinity adsorbent was more satisfactory.

In the present system, when the lower concentrations (10 $\mathrm{mg} \cdot \mathrm{L}^{-1}$ and $2.5 \mathrm{mg} \cdot \mathrm{L}^{-1}$ ) of the extract solution was tested for the recognition ability of the PcAb, better results were obtained but the running time for analysis were prolonged (about $4 \sim 6 \mathrm{~h}$ ) accordingly. It is not surprising that the frontal time is getting longer with the lower concentration of the analyte, and then the difference of the retardation between them is more obvi- ous as well as the recognition ability getting better. It could be expected that the application of PcAb for high-throughput screening of novel inhibitors would be efficient in both natural resources and synthetic combinatorial libraries, which would be very beneficial for the discovery of lead compounds and drug candidates.

\section{References}

1 Fahad, A. A. ; Jinzi, J. W. ; Kit, S. L. Biopolymers, 1998, 47: 197

2 Plowman, G. D. ; Ullrich, A. ; Shawver, L. K. Drug News Perspect, 1994, 7: 334

3 Salomon, D. S. ; Brandt, R. ; Ciadiello, F. ; Normanno, N. Crit. Rev. Oncol. Haematol., 1995, 19: 183

4 Gullick, W. J. Br. Med. Bull., 1991, 47: 87

5 Woodburn, J. R. Pharmacol. Ther., 1999, 82: 241

6 Levitzki, A. FASEB J., 1992, 6: 3275

7 Dobrusin, E. M. ; Fry, D. W. Annu. Rep. Med. Chem., 1992, 27 : 169

8 Zhu, L. L. ; Xu, X. J. J. Chromatogr. A, (In Press)

9 Fry, D. W. ; Kraker, A. J. ; McMichael, A. ; Ambroso, L. A. ; Nelson, J. M. ; Leopold, W. R. ; Connors, R. W. ; Bridges, A. J. Science, 1994, 265: 1093

\title{
利用多克隆抗体有效识别中草药中抗表皮生长因子抑制剂 *
}

\author{
朱丽荔徐符杰 \\ (北京大学化学与分子工程学院,北京 100871)
}

\begin{abstract}
摘要 用一种已知的抗表皮生长因子受体抑制剂 (piceatannol) 作为半抗原与载体牛血清白蛋白 (BSA) 连 接后免疫制备相应的多克隆抗体 (PcAb)。利用该多克隆抗体来模拟酶制成亲和色谱柱,从一种藏药粗提物 中将包括该半抗原在内的几种结构不同的抗表皮生长因子受体抑制剂识别出来. 研究采用前沿亲和色谱 质谱联用技术对样品进行分析, 可以直接从中药复杂体系中识别出有效成分并进行鉴定, 实现中药有效成 分的篮选与鉴定一体化技术.
\end{abstract}

关键词：表皮生长因子受体，多克隆抗体，抑制剂，前沿亲和色谱 - 质谱联用 中图分类号： O641 